\title{
Lift Force on a Circular Arc Wing
}

\section{Kern E. Kenyon}

4632 North Lane, Del Mar, USA

Correspondence to: Kern E. Kenyon, Kernken@aol.com

Keywords: Lift Force on a Wing

Received: September 3, $2017 \quad$ Accepted: September 26, $2017 \quad$ Published: September 29, 2017

Copyright $\odot 2017$ by authors and Scientific Research Publishing Inc.

This work is licensed under the Creative Commons Attribution International License (CC BY 4.0).

http://creativecommons.org/licenses/by/4.0/

\section{(c) (7) Open Access}

\section{ABSTRACT}

The lift force is calculated for a gliding wing with a circular arc top and a flat bottom in a uniform fluid. It is: const $\rho U^{2} / R_{0}$, where $\rho$ is the constant fluid density, $U$ is the uniform flow speed far from the wing and $R_{0}$ is the radius of curvature of the wing's top surface. To obtain this result two non-linear differential equations in pressure and velocity are combined into one linear governing equation for velocity, which contains a non-constant coefficient, $R(z)$, the radius of curvature of the streamlines above the wing as a function of the vertical coordinate $z$. Bernoulli's principle along a streamline and the force balance across a streamline (pressure gradient equals centrifugal force) are the starting equations. A solution to the governing equation is derived by providing an algebraic function for $R(z)$ that is consistent with observations, and the order of magnitude one constant in the lift force is worked out. It is believed that the present approach to understanding the lift force on a wing has not been tried before. More theoretical and observational work are needed to better specify $R(z)$.

\section{INTRODUCTION}

For more than a century, efforts have been made to understand the lift force on the gliding wing of a bird or plane using methods of mathematics and physics. Not so many years ago I made one myself [1], though that effort was limited to "thin" wings. Since practical applications are potentially so overwhelmingly important, every possible approach toward an increased understanding of this problem should be attempted. A different avenue is explored here and applied to a particularly simple wing shape for ease of illustration. Like all earlier studies the current approach has advantages and disadvantages. Being common to the available methods, including the one below, is the chief limitation: there is an incompleteness of the theory. This incompleteness is brought into a sharp focus in the present development, which could be useful for stimulating further work.

Not yet sufficiently appreciated, perhaps since apparently it's not immediately self-evident, is that the perturbed fluid velocity above the wing must die away with increasing distance from the solid surface suf- 
ficiently quickly, otherwise there will be very little lift. Then what exactly is the physics behind the die-off rate of the perturbed flow? In general, observations show that the perturbation's decrease in velocity with increase in distance is taking place, but detailed velocity (or pressure) profiles from measurements are apparently missing. More streak photographs would be helpful.

In one of his Notebooks, Leonardo da Vinci observed a rock in a stream and noticed that the speed of flow right next to the sides of the rock was faster than the mean speed of the stream, and he commented that the flow would rather do that than go over the top of the rock. Evidently the presence of a rock would not slow the whole stream down, so the net flux may remain constant.

\section{MODEL}

Consider a uniform steady flow of an incompressible fluid at a speed that is in the range of slow to moderate, and neglect friction and the acceleration of gravity. Within this flow is a two-dimensional cross-section of a rigid solid wing with a flat bottom and a circular arc top, oriented such that the flat bottom is parallel to the direction of the flow far away from the wing (zero angle of attack). No turbulence or eddy shedding occurs in the fluid due to the presence of the wing by assumption. It is desired to calculate the lift force on this wing.

First, two separate differential equations are brought together in (1) and (2); each one contains the same two variables: pressure and velocity. In a sense this is already a step in the right direction, compared to traditional potential flow theories, whereby velocities are computed directly from the Laplace equation but pressure information is entirely left out. For example, potential flow theory gives the perturbed flow past a cylinder diminishing away as the inverse square of the distance from the cylinder. Not only is pressure unavailable but the physics of this decay law goes unexplained, plus it is not even known if observations agree with the theory in this respect.

Along a streamline there is Bernoulli's Equation (1). Across a streamline there is a force balance (2), to maintain a steady state, between the upward centrifugal force on the curved flow above the wing and a downward pressure gradient.

Two governing equations for the pressure $p$ and velocity $V$ are:

$$
\begin{gathered}
p=\text { const }-\frac{1}{2} \rho V^{2} \\
\frac{\mathrm{d} p}{\mathrm{~d} z}=\rho \frac{V^{2}}{R}
\end{gathered}
$$

where $\rho$ is the constant density and $R$ is the non-constant radius of curvature of the streamlines, which is a function of $z$, the vertical coordinate. The constant in Bernoulli's Equation (1) is taken to be the same for all streamlines for simplicity.

To specify the geometry of the wing in cross-section it is sufficient to let the constant $R_{0}$ be the radius of curvature of the wing's top surface and $h$ be the maximum (vertical) thickness. There is no need a priori to assume a thin wing since the same model can be applied to flow across the top (or bottom) of a cylinder [2].

Although Equations (1) and (2) are two equations in two unknowns, each one by itself is nonlinear. Research could easily stop at this point; one might logically recoil at the nonlinear barrier. However, curiosity to continue on is rewarded by unexpectedly opening up a relatively simple way through the difficulty.

Eliminate one of the two variables between (1) and (2). It does not matter much which variable is eliminated first. Therefore, take the $z$ derivative of (1), combine that with (2), and get one equation in one variable, $V$.

$$
\frac{\mathrm{d} V}{\mathrm{~d} z}=-\frac{V}{R}
$$


which is a linear first order ordinary differential equation with a non-constant coefficient. Biggest surprise is that it is linear, and therefore solvable!

\section{SOLUTION}

A solution to (3) can be started easily enough by using elementary manipulations

$$
V=V_{0}\left(\mathrm{e}^{f(z)}-1\right)+U
$$

where

$$
f(z)=\int_{0}^{z} \frac{d z^{\prime}}{R\left(z^{\prime}\right)} d z
$$

And $V_{0}$ is a constant to be determined later by conserving mass between two vertical cross-sections, one on top of the wing and one far away from the wing. Also $U$ is the uniform flow speed at a great distance in any direction from the top of the wing. The vertical coordinate's origin is taken at the top of the wing, increasing upward with positive values.

To complete the solution of (4) information on the radius of curvature function $R(z)$ needs to be supplied to the equation, either from measurements or from another theory. This is the incompleteness of the present model. An algebraically convenient representation of the radius of curvature function is adopted until something better comes along

$$
R(z)=R_{0}\left(1+\frac{z}{R_{0}}\right)^{n}
$$

which qualitatively agrees with observations in the sense that as the distance away from the top of the wing increases, so the shape of the streamlines becomes flatter (less curved). If $n$ is an integer greater than or equal to 3, the constant in (4), $V_{0}$, can be determined by conservation of mass. However, when $n$ is 2 or less that constant cannot be found because the integrals involved with conserving mass do not converge. Thus one could infer that some information has just been obtained about the strength of the die-off rate of the perturbed flow over the top of the wing.

In what follows $n=3$ is chosen. When that exponent is put into (6) and (6) is inserted into (5), then (5) can be evaluated by a standard integration. With the exponent in (4) now evaluated and the exponential term approximated by a series of increasingly smaller terms (only three were kept), conservation of mass can be carried out to determine the constant in (4). That turns out to be

$$
V_{0}=2 U \frac{h}{R_{0}}
$$

All the pieces are at hand now for computing the lift force on the circular arc wing.

\section{LIFT FORCE}

Because the flow speed immediately above the wing is faster than that below the wing, the net Bernoulli effect is an upward lift force on the wing. Along the $z$-axis it is

$$
\text { Lift Force }=\frac{1}{2} \rho\left(\frac{V^{2}(0)-U^{2}}{h}\right) \approx 1.3 \frac{\rho U^{2}}{R_{0}}
$$

Another way to derive (8), which is every bit as convenient, starts by combining (1) and (2) into one equation in the pressure by eliminating the velocity. A very similar Equation to (3) is obtained in which pressure replaces velocity except there is a factor of two multiplying the RHS [3]. Then in the solution the factor of two translates into the exponent of the exponential term. This is significant because it means that 
the model predicts that the pressure perturbation decays more rapidly away from the top of the wing than the velocity perturbation does. Standard theories do not make such a claim, nor anything analogous to it.

Not to be taken as exact by any means is the numerical coefficient in (8), because it depends on the die-off rate of the radius of curvature function, which is not known even approximately. If the die-off rate is quicker than represented by (6) with $n=3$, then the lift will be larger than given by (8), since the speed at the top of the wing will be greater by conservation of mass.

Notice that the thickness of the wing does not appear in (8).

One way to go forward in the future is to make detailed measurements of the flow above the wing, possibly from streak photographs, from which the radius of curvature of the streamlines can be computed as a function of distance away from the wing's top surface. Then that information can be fed into the non-constant coefficient $R(z)$ and the linear differential governing equation for the velocity over the wing (3) will be easily integrated by computer. After that the lift force can be calculated as done above.

\section{CONCLUSION}

A new application of a recent fluid model is made to compute the lift force on an idealized wing shape. Two non-linear differential equations in pressure and velocity are combined into a single linear equation in velocity for flow over a wing cross-section with a circular arc top and a flat bottom. Though linear the governing equation has a non-constant coefficient: the radius of curvature of the streamlines, which increases with increasing distance from the top of the wing. When an algebraic form for the coefficient, consistent with observations, is supplied, the governing equation is solved and the lift force calculated. It is the fluid density times the square of the flow speed far from the wing divided by the radius of curvature of the wing's top surface. There is also a multiplying constant, of order of magnitude one, that depends on the particular formula selected for the radius of curvature of the streamlines above the wing. More work is needed to make this curvature function come closer to agreeing with measurements.

\section{REFERENCES}

1. Kenyon, K.E. (1989) Lift Theory for Level Flight Using Bernoulli's Principle and Cross-Stream Acceleration. Physics Essays, 2, 241-245. https://doi.org/10.4006/1.3035874

2. Kenyon, K.E. (2016) On the Magnus Effect. Natural Science, 8, 49-52. http://dx.doi.org/10.4236/ns.2016.82006

3. Kenyon, K.E. (2013) Flow Past a Cylinder. Journal of Scientific Theory and Methods, 2013, 211-222. 